\title{
Generalized pattern frequency in large permutations
}

\author{
Joshua Cooper * \\ Department of Mathematics \\ University of South Carolina \\ Columbia, SC, U.S.A. \\ cooper@math.sc.edu \\ Erik Lundberg \\ Department of Mathematics \\ Purdue University \\ West Lafayette, IN, U.S.A. \\ elundber@math.purdue.edu \\ Brendan Nagle ${ }^{\dagger}$ \\ Department of Mathematics and Statistics \\ University of South Florida \\ Tampa, FL, U.S.A. \\ bnagle@usf .edu
}

Submitted: Sep 7, 2012; Accepted: Jan 29, 2013; Published: Feb 5, 2013

Mathematics Subject Classifications: 05A05, 05A16

\begin{abstract}
In the study of permutations, generalized patterns extend classical patterns by adding the requirement that certain adjacent integers in a pattern must be adjacent in the permutation.

For any generalized pattern $\pi_{0}^{*}$ of length $k$ with $1 \leqslant b \leqslant k$ blocks, we prove that for all $\mu>0$, there exists $0<c=c(k, \mu)<1$ so that whenever $n \geqslant n_{0}(k, \mu, c)$, all but $c^{n} n$ ! many $\pi \in S_{n}$ admit $(1 \pm \mu) \frac{1}{k !}\left(\begin{array}{l}n \\ b\end{array}\right)$ occurrences of $\pi_{0}^{*}$. Up to the choice of $c$, this result is best possible for all $\pi_{0}^{*}$ with $k \geqslant 2$.

We also give a lower bound on avoidance of the generalized pattern 12-34, which answers a question of S. Elizalde [8] (2006).
\end{abstract}

Keywords: generalized patterns; pattern avoidance; Azuma's inequality; Chernoff's inequality; Sharkovsky's Theorem

\section{Introduction}

Pattern and generalized pattern avoidance in permutations is a well-studied area (see, e.g., $[1-5,7,8,10,11])$. Fix $1 \leqslant k \leqslant n$ and $\pi_{0} \in S_{k}$ and let $\pi \in S_{n}$. An occurrence

\footnotetext{
*The first author was partially supported by NSF grant DMS-1001370.
}

${ }^{\dagger}$ The third author was partially supported by NSF grant DMS-1001781 
of a pattern $\pi_{0}$ in $\pi$ is a sequence of integers $1 \leqslant \ell_{1}<\cdots<\ell_{k} \leqslant n$ so that, for all $1 \leqslant i \neq j \leqslant k$,

$$
\pi\left(\ell_{i}\right)<\pi\left(\ell_{j}\right) \quad \Longleftrightarrow \quad \pi_{0}(i)<\pi_{0}(j) .
$$

In order to define generalized patterns, take a classical pattern $\pi_{0}=\left(a_{1}, \ldots, a_{k}\right)=$ $\left(\pi_{0}(1), \ldots, \pi_{0}(k)\right)$, and fix $\pi_{0}^{*}=\left(a_{1}, \varepsilon_{1}, a_{2}, \varepsilon_{2}, \ldots, \varepsilon_{k-1}, a_{k}\right)$ where, for each $1 \leqslant i \leqslant k-1$, $\varepsilon_{i}$ is either a dash '-' or the empty string. Then, $\pi \in S_{n}$ admits $\pi_{0}^{*}$ as a generalized pattern if it contains an occurrence $1 \leqslant \ell_{1}<\cdots<\ell_{k} \leqslant n$ of the classical pattern $\pi_{0}$ satisfying that,

$$
\text { whenever } \varepsilon_{i} \neq- \text {, then } \ell_{i+1}=\ell_{i}+1 \text {. }
$$

More explicitly, suppose, for some positive integer sequence $\mathbf{q}=\left(q_{1}, \ldots, q_{b}\right)$, for which $q_{1}+\cdots+q_{b}=k$, that

$$
\begin{aligned}
\pi_{0}^{*}=\pi_{0}^{\mathbf{q}} & =\left(a_{1}, \ldots, a_{q_{1}},-, a_{q_{1}+1}, \ldots, a_{q_{1}+q_{2}},-, \ldots,-, a_{k-q_{b}+1}, \ldots, a_{k}\right) \\
& =\left(A_{1},-, A_{2},-, \ldots,-, A_{b}\right) .
\end{aligned}
$$

Then, for some integers $1 \leqslant \hat{\ell}_{1}<\cdots<\hat{\ell}_{b} \leqslant n$,

$$
\begin{aligned}
\left(\ell_{1}, \ldots, \ell_{k}\right) & =\left(\hat{\ell}_{1}, \ldots, \hat{\ell}_{1}+q_{1}-1, \hat{\ell}_{2}, \ldots, \hat{\ell}_{2}+q_{2}-1, \ldots, \hat{\ell}_{b}, \ldots, \hat{\ell}_{b}+q_{b}-1\right) \\
& =\left(L_{1}, \ldots, L_{b}\right) .
\end{aligned}
$$

We shall refer to the subsequences $A_{1}, \ldots, A_{b}$ and $L_{1}, \ldots, L_{b}$ as blocks.

As an illustrative example, we note that the permutation $(3,5,2,4,1)=35241$ contains the classical pattern 132 (realized uniquely by the 3,5 , and 4 occuring in that order). However, 35241 does not contain the generalized pattern 1-32, since the 5 and 4 are not adjacent.

Let $f_{\pi_{0}^{*}}(\pi)$ denote the frequency of the generalized pattern $\pi_{0}^{*}$ in $\pi$, and set $F_{\pi_{0}}(\pi)=$ $f_{\pi_{0}^{*}}(\pi)$ in the case that $\mathbf{q}=(1, \ldots, 1)$ (i.e., classical patterns). In this notation, the celebrated result of Marcus and Tardos [13] (cf. Klazar [11]) asserts $F_{\pi_{0}}(\pi) \geqslant 1$ for all but $C^{n}$ permutations $\pi \in S_{n}$, where $C=C\left(\pi_{0}\right)>1$ and $n$ is sufficiently large. The first author [7] proved that $F_{\pi_{0}}$ is concentrated about its mean: $F_{\pi_{0}}(\pi)=(1 \pm o(1)) \frac{1}{k !}\left(\begin{array}{l}n \\ k\end{array}\right)$ for all but $o(n !)$ permutations $\pi \in S_{n}$. Our main result shows, more generally, that $f_{\pi_{0}^{*}}$ is also concentrated about its mean, and we provide a sharp estimate for the error $o(n !)$ of concentration.

Theorem 1. For every $k \geqslant 1$ and for all $\mu>0$, there exists $0<c<1$ so that, for all sufficiently large integers $n$, the following holds. For every $\pi_{0} \in S_{k}$ and for every sequence $\pi_{0}^{*}$ with $b$ blocks as in (3), all but $c^{n} n !$ many $\pi \in S_{n}$ satisfy $f_{\pi_{0}^{*}}(\pi)=(1 \pm \mu) \frac{1}{k !}\left(\begin{array}{l}n \\ b\end{array}\right)$.

Remark 2. In Section 2, we offer two proofs of Theorem 1. The first, based on martingales, is fairly short. The second gives more detail, using a 'quasi-random' property (see Lemma 6) typical of random permutations. Lemma 6 extends some results from [7] and may be of independent interest.

Up to the choice of $0<c<1$, Theorem 1 is best possible for all $\pi_{0}^{*}$ with $k \geqslant 2$. In particular, we prove the following result. 
Proposition 3. Fix $k \geqslant 2, b \geqslant 1$, and $\pi_{0}=\left(a_{1}, \ldots, a_{k}\right) \in S_{k}$. Let $\pi_{0}^{*}$ be any sequence, as in (3), with $b$ blocks. Then, there exists $0<\gamma_{0}<1$ so that, for all $0<\gamma<\gamma_{0}$, there exist infinitely many integers $n$ for which at least $\gamma^{n} n$ ! permutations $\pi \in S_{n}$ satisfy $f_{\pi_{0}^{*}}(\pi)<\gamma n^{b}$.

We prove Proposition 3 in Section 3.

Proposition 3 can often be strengthened. Indeed, S. Elizalde [8] proved the following strong and quite general result (in [8], see Proposition 4.3).

Theorem 4 (Elizalde [8]). Let $\pi_{0}^{*}$ be a sequence, as in (3), having a block $A_{i}$ of length at least 3. Then, there exists $0<c<1$ so that for all $n \geqslant k$, at least $c^{n} n$ ! permutations $\pi \in S_{n}$ satisfy $f_{\pi_{0}^{*}}(\pi)=0$.

Elizalde [8] also considered to what extent Theorem 4 can be extended to sequences $\pi_{0}^{*}$ whose every block has length at most two. He showed that, in general, Theorem 4 can't be extended to every such $\pi_{0}^{*}$. To describe these results, let $A_{n}\left(\pi_{0}^{*}\right)$ denote the set of permutations $\pi \in S_{n}$ for which $f_{\pi_{0}^{*}}(\pi)=0$, and let $\alpha_{n}\left(\pi_{0}^{*}\right)=\left|A_{n}\left(\pi_{0}^{*}\right)\right|$. For $(1,-, 2,3,-, 4)=1-23-4$, Elizalde showed (see Corollary 6.2 in [8])

$$
\lim _{n \rightarrow \infty}\left(\frac{\alpha_{n}(1-23-4)}{n !}\right)^{1 / n}=0 .
$$

He asked (see Section 7 of [8]):

$$
\text { does } \lim _{n \rightarrow \infty}\left(\frac{\alpha_{n}(12-34)}{n !}\right)^{1 / n}=0 \text { ? }
$$

We answer this question in the negative.

Theorem 5. For odd integers n,

$$
\alpha_{n}(12-34) \geqslant\left(\frac{1}{2}-o(1)\right)^{n} n !
$$

We prove Theorem 5 in Section 4, and also consider some related problems.

\section{Proofs of Theorem 1}

For both of the following proofs, fix a positive integer $k$ and fix $\mu>0$.

\subsection{The martingale proof}

Let

$$
c=\exp \left\{-\frac{\mu^{2}}{9 k^{4} k !^{2}}\right\}
$$


and let $n$ be a sufficiently large integer wherever needed. Fix $\pi_{0}^{*}$ with $b$ blocks as in (3). We show that all but $c^{n} n$ ! many $\pi \in S_{n}$ satisfy $f_{\pi_{0}^{*}}(\pi)=(1 \pm \mu) \frac{1}{k !}\left(\begin{array}{l}n \\ b\end{array}\right)$.

To that end, let $\pi \in S_{n}$ be chosen uniformly at random. We use the 'exposure process' to define the following sequence of random variables. Set

$$
X_{0}=\mathbb{E}\left[f_{\pi_{0}^{*}}(\pi)\right] \text {, where from }(4) \text {, we have }(1-o(1)) \frac{1}{k !}\left(\begin{array}{l}
n \\
b
\end{array}\right) \leqslant \mathbb{E}\left[f_{\pi_{0}^{*}}(\pi)\right] \leqslant \frac{1}{k !}\left(\begin{array}{l}
n \\
b
\end{array}\right) .
$$

For $r \in[n]=\{1, \ldots, n\}$, let $\pi_{[r]}$ denote the restriction $\pi:[r] \rightarrow[n]$. Set

$$
X_{r}=\mathbb{E}\left[f_{\pi_{0}^{*}}(\pi) \mid \pi_{[r]}\right]
$$

so that $X_{n}=f_{\pi_{0}^{*}}(\pi)$ is the variable we wish to estimate. Then, $X_{0}, X_{1}, \ldots, X_{n}$ is the Doob martingale for the function $f_{\pi_{0}^{*}}$, to which we will apply Azuma's inequality.

For that purpose, observe that for each $0 \leqslant r \leqslant n-1$,

$$
\left|X_{r+1}-X_{r}\right| \leqslant k\left(\begin{array}{c}
n \\
b-1
\end{array}\right) \text {. }
$$

To see this, note that the element $r+1$ belongs to between zero and $k\left(\begin{array}{c}n \\ b-1\end{array}\right)$ occurrences $\left(\ell_{1}, \ldots, \ell_{k}\right)$ of $\pi_{0}^{*}$ in $\pi$. Indeed, if $\ell_{i}=r+1$ belongs to block $L_{i^{\prime}}$ (see $(4)$ ), then all of $L_{i^{\prime}}$ is determined by $r+1=\ell_{i}$ and $\mathbf{q}=\mathbf{q}\left(\pi_{0}^{*}\right)$. Thus, it remains to determine $L_{1}, \ldots, L_{i^{\prime}-1}, L_{i^{\prime}+1}, \ldots, L_{b}$, or equivalently, $\hat{\ell}_{1}, \ldots, \hat{\ell}_{i^{\prime}-1}, \hat{\ell}_{i^{\prime}+1}, \ldots, \hat{\ell}_{b}$, of which there are at most $\left(\begin{array}{c}n \\ b-1\end{array}\right)$.

Applying Azuma's inequality with $t=(\mu / 2) X_{0}$ and using (8) and (9), we have

$$
\begin{aligned}
& \mathbb{P}\left[\left|X_{n}-X_{0}\right| \geqslant t\right] \leqslant 2 \exp \left\{-\frac{t^{2}}{2 \sum_{r=0}^{n-1}\left(X_{r+1}-X_{r}\right)^{2}}\right\} \leqslant \exp \left\{-\frac{\mu^{2} \frac{1}{k !^{2}}\left(\begin{array}{l}
n \\
b
\end{array}\right)^{2}}{8 n k^{2}\left(\begin{array}{c}
n \\
b-1
\end{array}\right)^{2}}(1-o(1))\right\} \\
& =\exp \left\{-\frac{\mu^{2} n}{8 k !^{2} k^{2} b^{2}}(1-o(1))\right\} \leqslant \exp \left\{-\frac{\mu^{2} n}{8 k !^{2} k^{4}}(1-o(1))\right\} \leqslant \exp \left\{-\frac{\mu^{2} n}{9 k !^{2} k^{4}}\right\} \stackrel{(7)}{=} c^{n} .
\end{aligned}
$$

Thus, with probability $1-c^{n}$,

$$
f_{\pi_{0}^{*}}(\pi)=\left(1 \pm \frac{\mu}{2}\right) \mathbb{E}\left[f_{\pi_{0}^{*}}(\pi)\right] \stackrel{(8)}{=}\left(1 \pm \frac{\mu}{2}\right)(1 \pm o(1)) \frac{1}{k !}\left(\begin{array}{c}
n \\
b
\end{array}\right)=(1 \pm \mu) \frac{1}{k !}\left(\begin{array}{c}
n \\
b
\end{array}\right)
$$

as desired.

\subsection{The quasi-random proof}

To present Lemma 6, we need a few concepts. For integers $n>t \geqslant j \geqslant 1$, define $I_{j}=[(j-1)\lfloor n / t\rfloor+1, j\lfloor n / t\rfloor]$ and $R=[n] \backslash \bigcup_{j=1}^{t} I_{j}$. We call $[n]=I_{1} \cup I_{2} \cup \cdots \cup I_{t} \cup R$ the $t$-partition $\boldsymbol{P}_{t}$ of $[n]$. Now, fix $\pi \in S_{n}$, and consider partitions $\boldsymbol{P}_{s}=I_{1} \cup \cdots \cup I_{s} \cup R_{s}$ and $\boldsymbol{P}_{t}=E_{1} \cup \cdots \cup E_{t} \cup R_{t}$ of $[n]$, where $n>t \geqslant s \geqslant q \geqslant 1$. For a set $X$, we will write 
$(X)_{m}$ for the family of $m$-permutations of $X$, and we write $(|X|)_{m}$ for $\left|(X)_{m}\right|$, when $|X|$ is finite. For $\mathbf{i}=\left(i_{1}, \ldots, i_{q}\right) \in([s])_{q}$ and $j \in[t]$, let

$$
E_{\mathbf{i} j}(\pi)=\left\{\hat{\ell} \in E_{j}: \hat{\ell}+q-1 \in E_{j} \text { and } \pi(\hat{\ell}+m-1) \in I_{i_{m}} \text { for all } m \in\{1, \ldots, q\}\right\} .
$$

For $\zeta>0$, and $(\mathbf{i}, j) \in([s])_{q} \times[t]$, we say $\pi \in S_{n}$ is $(\mathbf{i}, j, \zeta, q)$-typical (w.r.t. $\left.\left(\boldsymbol{P}_{s}, \boldsymbol{P}_{t}\right)\right)$ if

$$
\left|E_{\mathbf{i} j}(\pi)\right| \geqslant(1-\zeta) \frac{1}{(s)_{q}}\left|E_{j}\right|=(1-\zeta) \frac{1}{(s)_{q}}\left\lfloor\frac{n}{t}\right\rfloor
$$

and say $\pi \in S_{n}$ is $(\zeta, q)$-typical (w.r.t. $\left.\left(\boldsymbol{P}_{s}, \boldsymbol{P}_{t}\right)\right)$ if it is $(\mathbf{i}, j, \zeta, q)$-typical for all $(\mathbf{i}, j) \in$ $([s])_{q} \times[t]$.

Lemma 6. For all $\zeta>0$ and integers $q \geqslant 1$, there exists an integer $s_{0}$ so that for all integers $s \geqslant s_{0}$, there exists an integer $t_{0}$ so that for all integers $t \geqslant t_{0}$, there exists $c_{0}>0$ so that for all sufficiently large integers $n$, all but $\exp \left\{-c_{0} n\right\} n !$ permutations $\pi \in S_{n}$ are $(\zeta, q)$-typical w.r.t. $\left(\boldsymbol{P}_{s}, \boldsymbol{P}_{t}\right)$.

Lemma 6 follows by a standard (albeit tedious) probablistic analysis, which we give in Section 5 .

To show that Lemma 6 implies Theorem 1, define auxiliary constants $\delta, \zeta>0$ so that

$$
\delta=\frac{\mu}{k !} \quad \text { and } \quad(1-2 \zeta)^{k+2}>1-\delta .
$$

For $q \in[k]$, let $s_{0}(q)$ be the constant guaranteed by Lemma 6. Fix an integer $s$ so that

$$
s \geqslant \max \left\{s_{0}(1), \ldots, s_{0}(k)\right\} \quad \text { and } \quad\left(\begin{array}{l}
s \\
k
\end{array}\right) \geqslant \frac{s^{k}}{k !}(1-2 \zeta) .
$$

For $q \in[k]$, let $t_{0}(q)$ be the constant guaranteed by Lemma 6. Fix an integer $t$ with

$$
t \geqslant \max \left\{t_{0}(1), \ldots, t_{0}(k)\right\} \quad \text { and so that for all } b \in[k], \quad\left(\begin{array}{l}
t \\
b
\end{array}\right) \geqslant \frac{t^{b}}{b !}(1-2 \zeta) .
$$

For $q \in\{1, \ldots, k\}$, let $c_{0}(q)>0$ be the constant guaranteed by Lemma 6 . Define

$$
c_{0}=\min \left\{c_{0}(1), \ldots, c_{0}(k)\right\} \quad \text { and } \quad c=\exp \left\{-c_{0} / 4\right\} .
$$

In all that follows, let $n$ be a sufficiently large integer.

Fix a permutation $\pi_{0} \in S_{k}$, and let $\pi_{0}^{*}=\pi_{0}^{\mathbf{q}}=\left(A_{1},-, \ldots,-, A_{b}\right)$ be given as in (3) where $\mathbf{q}=\left(q_{1}, \ldots, q_{b}\right)$. Apply Lemma 6 (cf. (13)-(15)) to conclude that all but

$$
\left(\exp \left\{-c_{0}\left(q_{1}\right) n\right\}+\cdots+\exp \left\{-c_{0}\left(q_{b}\right) n\right\}\right) n ! \leqslant k \exp \left\{-c_{0} n\right\} n ! \leqslant \exp \left\{-\frac{c_{0}}{2} n\right\} n !
$$

permutations $\pi \in S_{n}$ are $\left(\zeta, q_{x}\right)$-typical w.r.t. $\left(\boldsymbol{P}_{s}, \boldsymbol{P}_{t}\right)$ for all $x \in[b]$. For such a $\pi \in S_{n}$, we show

$$
f_{\pi_{0}^{\mathrm{q}}}(\pi) \geqslant(1-\delta) \frac{1}{k !}\left(\begin{array}{c}
n \\
b
\end{array}\right) \stackrel{(12)}{>}(1-\mu) \frac{1}{k !}\left(\begin{array}{l}
n \\
b
\end{array}\right) .
$$


Indeed, fix indices $1 \leqslant i_{1}<\cdots<i_{k} \leqslant s$ and $1 \leqslant j_{1}<\cdots<j_{b} \leqslant t$. For $x \in[b]$, recall the block

$$
A_{x}=\left(a_{q_{1}+\cdots+q_{x-1}+1}, \ldots, a_{q_{1}+\cdots+q_{x}}\right)=\left(\pi_{0}\left(q_{1}+\cdots+q_{x-1}+1\right), \ldots, \pi_{0}\left(q_{1}+\cdots+q_{x}\right)\right)
$$

of $\pi_{0}^{\mathbf{q}}$ (cf. (3)). Consider the injection defined by, for each $x \in[b]$,

$$
\begin{aligned}
j_{x} \longmapsto \mathbf{i}_{x} \stackrel{\text { def }}{=}\left(i_{a}\right)_{a \in A_{x}} & =\left(i_{a_{q_{1}+\cdots+q_{x-1}+1}}, \ldots, i_{a_{q_{1}+\cdots+q_{x}}}\right) \\
& =\left(i_{\pi_{0}\left(q_{1}+\cdots+q_{x-1}+1\right)}, \ldots, i_{\pi_{0}\left(q_{1}+\cdots+q_{x}\right)}\right) .
\end{aligned}
$$

For each $x \in[b]$, arbitrarily select $\hat{\ell}_{x} \in E_{\mathbf{i}_{x} j_{x}}(\pi)$ (cf. (10)). We claim that the sequence

$$
\left(L_{1}, L_{2}, \ldots, L_{b}\right), \quad \text { where for each } x \in[b], \quad L_{x}=\left(\hat{\ell}_{x}, \hat{\ell}_{x}+1, \ldots, \hat{\ell}_{x}+q_{x}-1\right),
$$

is exactly an occurrence in $\pi$ of the generalized pattern $\pi_{0}^{\mathbf{q}}$. The sequence $\left(L_{1}, \ldots, L_{b}\right)$ clearly satisfies (2), since each $L_{m}$ is consecutive, and since $\left(L_{1}, L_{2}, \ldots, L_{b}\right)$ precisely mimics the block structure of $\pi_{0}^{\mathbf{q}}=\left(A_{1},-, A_{2},-, \ldots,-, A_{b}\right)$ (cf. (3)). It remains to check, therefore, that $\left(L_{1}, \ldots, L_{b}\right)$ is an occurrence of the classical pattern $\pi_{0}$ in $\pi$, i.e., that $\left(L_{1}, \ldots, L_{b}\right)$ satisfies $(1)$.

Indeed, rewrite the sequence $\left(L_{1}, \ldots, L_{b}\right)$ as

$$
\begin{aligned}
& \left(\ell_{1}, \ldots, \ell_{k}\right)=\left(\ell_{1}, \ldots, \ell_{q_{1}}, \ell_{q_{1}+1}, \ldots, \ell_{q_{1}+q_{2}}, \ldots, \ell_{k-q_{b}+1}, \ldots, \ell_{k}\right) \\
& \quad \text { so that for } x \in[b], \quad L_{x}=\left(\ell_{q_{1}+\cdots+q_{x-1}+1}, \ldots, \ell_{q_{1}+\cdots+q_{x}}\right) .
\end{aligned}
$$

Comparing (19) and (20), we see that a term of the sequence $\left(L_{1}, \ldots, L_{b}\right)$ is determined by a choice of indices $1 \leqslant x \leqslant b$ and $1 \leqslant w \leqslant q_{x}$, and written simultaneously as

$$
\hat{\ell}_{x}+w-1=\ell_{q_{1}+\cdots+q_{x-1}+w} .
$$

(Such a term necessarily belongs to the block $L_{x}$.) Observe from (10) and (17) that

$$
\pi\left(\hat{\ell}_{x}+w-1\right) \in I_{i(x, w)}, \quad \text { where } \quad i(x, w)=i_{\pi_{0}\left(q_{1}+\cdots+q_{x-1}+w\right)} .
$$

Now, fix two terms (cf. (21)) of the sequence $\left(L_{1}, \ldots, L_{b}\right)$ :

$$
\hat{\ell}_{x}+w-1=\ell_{q_{1}+\cdots+q_{x-1}+w} \quad \text { and } \quad \hat{\ell}_{y}+z-1=\ell_{q_{1}+\cdots+q_{y-1}+z},
$$

where $1 \leqslant x, y \leqslant b, 1 \leqslant w \leqslant q_{x}$ and $1 \leqslant z \leqslant q_{y}$. From (22), we conclude

$$
\begin{aligned}
& \pi\left(\ell_{q_{1}+\cdots+q_{x-1}+w}\right)<\pi\left(\ell_{q_{1}+\cdots+q_{y-1}+z}\right) \Longleftrightarrow \max I_{i(x, w)}<\min I_{i(y, z)} \\
& \Longleftrightarrow i(x, w)<i(y, z) \stackrel{(22)}{\Longleftrightarrow} i_{\pi_{0}\left(q_{1}+\cdots+q_{x-1}+w\right)}<i_{\pi_{0}\left(q_{1}+\cdots+q_{y-1}+z\right)} \\
& \Longleftrightarrow \pi_{0}\left(q_{1}+\cdots+q_{x-1}+w\right)<\pi_{0}\left(q_{1}+\cdots+q_{y-1}+z\right),
\end{aligned}
$$

as required by (1). (For the last step, recall the ordering $1 \leqslant i_{1}<\cdots<i_{k} \leqslant s$ of the fixed indices.) 
Now, the discussion above implies that

$$
f_{\pi_{0}^{\mathbf{q}}}(\pi) \geqslant \sum \sum\left\{\prod_{x=1}^{b}\left|E_{\mathbf{i}_{x} j_{x}}(\pi)\right|: 1 \leqslant i_{1}<\cdots<i_{k} \leqslant s, 1 \leqslant j_{1}<\cdots<j_{b} \leqslant t\right\} .
$$

Since $\pi \in S_{n}$ is $(\zeta, q)$-typical w.r.t. $\left(\boldsymbol{P}_{s}, \boldsymbol{P}_{t}\right)$ for every $q \in\left\{q_{1}, \ldots, q_{b}\right\}$, we have, for each $x \in[b]$,

$$
\left|E_{\mathbf{i}_{x} j_{x}}(\pi)\right| \geqslant(1-\zeta) \frac{1}{(s)_{q_{x}}}\left\lfloor\frac{n}{t}\right\rfloor \geqslant(1-2 \zeta) \frac{n}{t(s)_{q_{x}}} \geqslant(1-2 \zeta) \frac{n}{t s^{q_{x}}} .
$$

Returning to (23),

$$
\begin{aligned}
& \frac{f_{\pi_{0}^{\mathbf{q}}(\pi)}\left(\begin{array}{l}
s \\
k
\end{array}\right)\left(\begin{array}{l}
t \\
b
\end{array}\right)}{2} \geqslant(1-2 \zeta)^{b}\left(\frac{n}{t}\right)^{b} \prod_{x=1}^{b} \frac{1}{s^{q_{x}}}=(1-2 \zeta)^{b}\left(\frac{n}{t}\right)^{b} \frac{1}{s^{q_{1}+\cdots+q_{b}}} \\
& =(1-2 \zeta)^{b}\left(\frac{n}{t}\right)^{b} \frac{1}{s^{k}} \geqslant(1-2 \zeta)^{k}\left(\frac{n}{t}\right)^{b} \frac{1}{s^{k}},
\end{aligned}
$$

and so (16) follows from

$$
f_{\pi_{0}^{\mathbf{q}}}(\pi) \geqslant\left(\begin{array}{l}
s \\
k
\end{array}\right)\left(\begin{array}{l}
t \\
b
\end{array}\right)(1-2 \zeta)^{k}\left(\frac{n}{t}\right)^{b} \frac{1}{s^{k}} \stackrel{(13),(14)}{\geqslant}(1-2 \zeta)^{k+2} \frac{1}{k !}\left(\frac{n^{b}}{b !}\right) \stackrel{(12)}{\geqslant}(1-\delta) \frac{1}{k !}\left(\begin{array}{l}
n \\
b
\end{array}\right) .
$$

The corresponding upper bound $f_{\pi_{0}^{\mathrm{q}}}(\pi) \leqslant(1+\mu) \frac{1}{k !}\left(\begin{array}{l}n \\ b\end{array}\right)$ follows, in fact, from the lower bound. Indeed, first conclude (16) for every permutation $p \in S_{k}$ and $p^{*}=p^{\mathbf{q}}$. Thus, all but

$$
k ! \exp \left\{-\frac{c_{0}}{2} n\right\} n !<\exp \left\{-\frac{c_{0}}{4} n\right\} n ! \stackrel{(15)}{=} c_{1}^{n} n !
$$

permutations $\pi \in S_{n}$ satisfy, for every $p \in S_{k}, f_{p^{\mathbf{q}}}(\pi) \geqslant(1-\delta) \frac{1}{k !}\left(\begin{array}{l}n \\ b\end{array}\right)$. Fix such a $\pi \in S_{n}$. Observe that every $1 \leqslant \ell_{1}<\cdots<\ell_{k} \leqslant n$ of the form in (19) and (20) defines a generalized pattern $p^{\mathbf{q}}$ of some $p \in S_{k}$. (Indeed, if $\pi\left(\left\{\ell_{1}, \ldots, \ell_{k}\right\}\right)=\left\{\lambda_{1}, \ldots, \lambda_{k}\right\}$, define $p(i)=j$ if and only if $\pi\left(\ell_{i}\right)=\lambda_{j}$.) Thus,

$$
\begin{aligned}
\left(\begin{array}{l}
n \\
b
\end{array}\right) \geqslant & \sum_{p \in S_{k}} f_{p^{\mathbf{q}}}(\pi)=f_{\pi_{0}^{\mathbf{q}}}(\pi)+\sum_{\pi_{0} \neq p \in S_{k}} f_{p^{\mathbf{q}}}(\pi) \geqslant f_{\pi_{0}^{\mathbf{q}}}(\pi)+(k !-1)(1-\delta) \frac{1}{k !}\left(\begin{array}{l}
n \\
b
\end{array}\right) \\
& \Longrightarrow \quad f_{\pi_{0}^{\mathbf{q}}}(\pi) \leqslant\left(\frac{1}{k !}+\delta-\frac{\delta}{k !}\right)\left(\begin{array}{l}
n \\
b
\end{array}\right) \leqslant(1+\delta k !) \frac{1}{k !}\left(\begin{array}{l}
n \\
b
\end{array}\right) \stackrel{(12)}{=}(1+\mu) \frac{1}{k !}\left(\begin{array}{l}
n \\
b
\end{array}\right) .
\end{aligned}
$$

\section{Proof of Proposition 3}

Fix $k \geqslant 2, b \geqslant 1$, and $\pi_{0}=\left(a_{1}, \ldots, a_{k}\right) \in S_{k}$. Fix any sequence $\pi_{0}^{*}$, as in (3), with $b$ blocks. If $\pi_{0}^{*}$ has a block of length at least 3 , then let $0<c=c\left(\pi_{0}^{*}\right)<1$ be the constant guaranteed by Theorem 4 , and set $\gamma_{0}=c / 2$. Otherwise, set $\gamma_{0}=1 / 2$. Fix $0<\gamma<\gamma_{0}$, and write $g=\lfloor 1 / \gamma\rfloor$, where we note that $\gamma<1 / 2$ implies $g \geqslant 2$. For a sufficiently large 
integer $n$ which is divisible by $g$, we guarantee at least $\gamma^{n} n$ ! permutations $\pi \in S_{n}$ with $f_{\pi_{0}^{*}}(\pi)<\gamma n^{b}$.

Our proof is based on cases, depending on the structure of the sequence $\pi_{0}^{*}$. Clearly, we get the following case entirely for free on account of Theorem 4.

Case $0\left(\pi_{0}^{*}\right.$ has a block of length at least 3$)$. Theorem 4 guarantees at least $c^{n} n !>\gamma^{n} n$ ! permutations $\pi \in S_{n}$ with $f_{\pi_{0}^{*}}(\pi)=0<\gamma n^{b}$.

To handle all other cases, we require the following considerations. For $0 \leqslant s \leqslant g-1$, write $I_{s}=[s(n / g)+1,(s+1)(n / g)]$ and $R_{s}=\{m \in[n]: m \equiv s(\bmod g)\}$. Then $[n]=I_{0} \cup \cdots \cup I_{g-1}$ and $[n]=R_{0} \cup \cdots \cup R_{g-1}$ are partitions of $[n]$ into parts of common size $n / g$. Consider the following four classes of permutations:

$$
\begin{array}{lll}
S_{n, 1}=\left\{\pi \in S_{n}: \pi\left(I_{s}\right)=I_{s}, \forall 0 \leqslant s \leqslant g-1\right\}, & \\
S_{n, 2}=\left\{\pi \in S_{n}: \pi\left(I_{s}\right)=I_{g-1-s}, \forall 0 \leqslant s \leqslant g-1\right\}, & \\
S_{n, 3}=\left\{\pi \in S_{n}: \pi\left(R_{s}\right)=I_{g-s}, \forall 0 \leqslant s \leqslant g-1\right\} & \text { (take } \left.I_{g}=I_{0}\right), \\
S_{n, 4}=\left\{\pi \in S_{n}: \pi\left(R_{s}\right)=I_{s-1}, \forall 0 \leqslant s \leqslant g-1\right\} & \text { (take } \left.I_{-1}=I_{g-1}\right) .
\end{array}
$$

Clearly, $\left|S_{n, 1}\right|=\left|S_{n, 2}\right|=\left|S_{n, 3}\right|=\left|S_{n, 4}\right|=((n / g) !)^{g}$, where by Stirling's formula,

$$
\left(\left(\frac{n}{g}\right) !\right)^{g}>\frac{1}{2}\left(\sqrt{2 \pi(n / g)}\left(\frac{n}{e g}\right)^{(n / g)}\right)^{g}>\frac{\sqrt{\gamma}}{2}(2 \pi \gamma n)^{\frac{g-1}{2}} \times \gamma^{n} \sqrt{2 \pi n}\left(\frac{n}{e}\right)^{n}>\gamma^{n} n ! .
$$

We mention, in advance, that in the following four cases below, Case $i$ will be handled by the family $S_{n, i}$, for $1 \leqslant i \leqslant 4$. We also mention that Cases 1 and 2 are not always disjoint from Case 0, nor are they always disjoint from each other. (It seemed easiest to preserve generality in the cases.)

We now consider when $\pi_{0}^{*}$ has $b \geqslant 2$ blocks. In particular, suppose $a_{i}=\pi_{0}(i)$ and $a_{j}=\pi_{0}(j), 1 \leqslant i<j \leqslant k$, belong to blocks $A_{i^{\prime}}$ and $A_{j^{\prime}}$, respectively, where $A_{i^{\prime}} \neq A_{j^{\prime}}$.

Case $1\left(b \geqslant 2, a_{i}>a_{j}\right)$. Fix $\pi \in S_{n, 1}$, and consider an occurence $1 \leqslant \ell_{1}<\cdots<\ell_{k} \leqslant n$ of the generalized pattern $\pi_{0}^{*}$ in $\pi$. Consider the terms $\ell_{i}<\ell_{j}$. From $(1)$, since $\pi_{0}(i)=$ $a_{i}>a_{j}=\pi_{0}(j)$, we have $\pi\left(\ell_{i}\right)>\pi\left(\ell_{j}\right)$. We therefore claim that, for some $1 \leqslant s \leqslant g$, we have $\ell_{i}, \ell_{j} \in I_{s}$. Indeed, if $\ell_{i} \in I_{s_{i}}$ and $\ell_{j} \in I_{s_{j}}$ for some $s_{i}<s_{j}$, then $\pi\left(\ell_{i}\right)<\pi\left(\ell_{j}\right)$ on account of $\pi \in S_{n, 1}$, a contradiction. We also recall from (3) and (4), that $\ell_{i}$ belongs to block $L_{i^{\prime}}$ and $\ell_{j}$ belongs to block $L_{j^{\prime}}$ (since $a_{i}$ belongs to block $A_{i^{\prime}}$ and $a_{j}$ belongs to block $\left.A_{j^{\prime}}\right)$. Finally, recall from (4) that $L_{i^{\prime}}$ begins with $\hat{\ell}_{i^{\prime}}$ and $L_{j^{\prime}}$ begins with $\hat{\ell}_{j^{\prime}}$. Then, since $\ell_{i}, \ell_{j} \in I_{s}$, we have that $\hat{\ell}_{j^{\prime}} \in I_{s}$ and $\hat{\ell}_{i^{\prime}} \in I_{s-1} \cup I_{s}$. (If $\hat{\ell}_{i^{\prime}} \in I_{s-1}$, it occurs very near the right boundary.) Clearly, there are at most $\left|I_{s}\right|=n / g$ choices for $\hat{\ell}_{j^{\prime}}$. It is easy to check that there are fewer than $n / g$ choices for $\hat{\ell}_{i^{\prime}}$. Clearly, there are at most $n^{b-2}$ choices for any remaining $\hat{\ell}_{1}, \ldots, \hat{\ell}_{b}$ in (4). Thus, $f_{\pi_{0}^{*}}(\pi)<n^{b-2} \sum_{s=1}^{g}(n / g)^{2} \leqslant \gamma n^{b}$.

Case $2\left(b \geqslant 2, a_{i}<a_{j}\right)$. Fix $\pi \in S_{n, 2}$. All details of Case 1 are repeated identically save the following: Now, $\pi\left(\ell_{i}\right)<\pi\left(\ell_{j}\right)$, which similarly implies that $\ell_{i}, \ell_{j} \in I_{s}$ for some $1 \leqslant s \leqslant g$. Indeed, $\ell_{i} \in I_{s_{i}}$ and $\ell_{j} \in I_{s_{j}}$ for some $s_{i}<s_{j}$ would imply $\pi\left(\ell_{i}\right)>\pi\left(\ell_{j}\right)$, on account of $\pi \in S_{n, 2}$. 
The only cases in the proof of Proposition 3 not covered by Cases 1 and 2 involve generalized patterns $\pi_{0}^{*}$ with $b=1$ block. (These are relatively rare, since there are only $k$ ! such, while there are $2^{k-1} k$ ! generalized patterns of $[k]$.) If $k \geqslant 3$ and $b=1$, then $\pi_{0}^{*}$ has (is) a block of length at least 3, which is included in Case 0. If $k=2$ and $b=1$, then $\pi_{0}^{*}=12$ or $\pi_{0}^{*}=21$, where these cases are entirely symmetric.

Case $3\left(\pi_{0}^{*}=12\right)$. Fix $\pi \in S_{n, 3}$, and consider an occurence $1 \leqslant \ell<\ell+1 \leqslant n$ of the generalized pattern 12 in $\pi$. From (1), we have that $\pi(\ell)<\pi(\ell+1)$. As such, $\pi \in S_{n, 3}$ implies that $\ell \equiv 0(\bmod g)$. Consequently, we have only $n / g \leqslant \gamma n$ choices for $\ell$.

Case $4\left(\pi_{0}^{*}=21\right)$. Fix $\pi \in S_{n, 4}$. An occurence $1 \leqslant \ell<\ell+1 \leqslant n$ of 21 in $\pi$ results in $\pi(\ell)>\pi(\ell+1)$. Since $\pi \in S_{n, 4}$, it must be that $\ell \equiv 0(\bmod g)$, resulting in only $n / g \leqslant \gamma n$ choices for $\ell$.

\section{Proof of Theorem 5}

Consider the following concept, which has a clear resemblance to patterns. For $\pi \in S_{n}$, call a pair $1<i<j<n$ a stretching pair if $\pi(i)<i<j<\pi(j)$. We shall use stretching pairs to prove Theorem 5, although stretching pairs are interesting in their own right, as we discuss in Section 4.2.

\subsection{Stretching pairs and Theorem 5}

We establish a few initial considerations. First, let $C_{n+1} \subset S_{n+1}$ denote the set of $(n+1)$ cycles of $S_{n+1}$, and write each $\pi \in C_{n+1}$ in cyclic notation: $\pi=\left(\begin{array}{llll}n+1 & a_{1} \ldots & a_{n}\end{array}\right)$, i.e., $\pi\left(a_{i}\right)=a_{i+1}$ for $0 \leqslant i \leqslant n$ and $a_{0}=a_{n+1}=n+1$. Consider the bijection $\phi: C_{n+1} \rightarrow S_{n}$ given by, for each $\pi=\left(n+1 a_{1} \ldots a_{n}\right) \in C_{n+1}$,

$$
p=\phi(\pi)=\left(a_{1}, \ldots, a_{n}\right) \text {, that is, } p(i)=a_{i} \text { for each } 1 \leqslant i \leqslant n .
$$

We prove that

$$
\begin{aligned}
\pi \in C_{n+1} & \text { admits a stretching pair } 1 \leqslant \pi(i)<i<j<\pi(j) \neq n+1 \\
& \text { if and only if } p=\phi(\pi) \text { admits } 21-34 \text { or } 34-21 \text { as a generalized pattern. }
\end{aligned}
$$

Before we prove (25), we note that $21-34$ is not the same as $12-34$, which Theorem 5 considers. However, Elizalde proved (see Proposition 5.3 from [8]) that

$$
\alpha_{n}(12-34)=\alpha_{n}(21-34)
$$

and so we shall be able to use (25).

Proof of (25). Suppose first that $p=f(\pi)=\left(a_{1}, \ldots, a_{n}\right) \in S_{n}$ admits $21-34$ or $34-21$ as a generalized pattern. If $a_{k}, a_{k+1}, a_{\ell}, a_{\ell+1}$ is a copy of 21-34, where $1<k+1<\ell<n$, then $a_{k+1}<a_{k}<a_{\ell}<a_{\ell+1}$, and so $\pi(i)=a_{k+1}<a_{k}=i<j=a_{\ell}<a_{\ell+1}=\pi(j) \leqslant n$ is a 
stretching pair of $\pi$. If $a_{k}, a_{k+1}, a_{\ell}, a_{\ell+1}$ is a copy of 34-21, then $a_{\ell+1}<a_{\ell}<a_{k}<a_{k+1}$, and so $\pi(i)=a_{\ell+1}<a_{\ell}=i<j=a_{k}<a_{k+1}=\pi(j) \leqslant n$ is a stretching pair of $\pi$. Assume now that $\pi=\left(\begin{array}{llll}n+1 & a_{1} & \ldots & a_{n}\end{array}\right) \in C_{n+1}$ admits a stretching pair $1 \leqslant \pi(i)<i<j<\pi(j) \leqslant n$. If $\pi=\left(\begin{array}{lllll}n+1 & a_{1} \ldots i \pi(i) \ldots j \pi(j) \ldots a_{n}\end{array}\right)$, then for some $1<k+1<\ell<n, p=f(\pi)$ has $i=a_{k}, \pi(i)=a_{k+1}, j=a_{\ell}$ and $\pi(j)=a_{\ell+1}$, where $a_{k+1}<a_{k}<a_{\ell}<a_{\ell+1}$ gives a copy of 21-34. If $\pi=\left(n+1 a_{1} \ldots j \pi(j) \ldots i \pi(i) \ldots a_{n}\right)$, then for some $1<k+1<\ell<n$, $p=f(\pi)$ has $j=a_{k}, \pi(j)=a_{k+1}, i=a_{\ell}$ and $\pi(i)=a_{\ell+1}$, where $a_{\ell+1}<a_{\ell}<a_{k}<a_{k+1}$ gives a copy of $34-21$.

Now, define $S_{n+1}^{\prime}$ to be the family of $\pi \in S_{n+1}$ satisfying $(n+1) / 2<\pi(i) \leqslant n+1$ if, and only if, $1 \leqslant i \leqslant(n+1) / 2$. Clearly, $S_{n+1}^{\prime}$ admits no stretching pairs. Set $C_{n+1}^{\prime}=$ $C_{n+1} \cap S_{n+1}^{\prime}$, and observe that $C_{n+1}^{\prime} \neq \emptyset$ if, and only if, $n$ is odd. As such, if $n$ is both odd and sufficiently large, Stirling's formula implies

$$
\left|C_{n+1}^{\prime}\right|=\frac{2}{n+1}\left(\left(\frac{n+1}{2}\right) !\right)^{2} \geqslant\left(\frac{1}{2}-o(1)\right)^{n} n !
$$

It then follows from (25) that $\phi\left(C_{n+1}^{\prime}\right)$ avoids $21-34$ and $34-21$, and so

$$
\begin{aligned}
\alpha_{n}(12-34) \stackrel{(26)}{=} \alpha_{n}(21-34) & \geqslant\left|A_{n}(21-34) \cap A_{n}(34-21)\right| \\
& \geqslant\left|\phi\left(C_{n+1}^{\prime}\right)\right|=\left|C_{n+1}^{\prime}\right| \geqslant\left(\frac{1}{2}-o(1)\right)^{n} n !
\end{aligned}
$$

which proves Theorem 5 .

\subsection{A corollary of Theorem 1 for stretching pairs}

Stretching pairs are motivated by considerations in dynamical systems. Namely, the occurrence of a stretching pair within a periodic orbit of a continuous interval map implies what is called 'turbulence' (see [3, 12] for details). These considerations are closely related to the celebrated theorem of Sharkovsky [14]. From this point of view, the second author [12] considered which $n$-cycles $\pi \in C_{n}$ admit stretching pairs, and proved that all but $o(n-1)$ ! of them do. Theorem 1 allows us to sharpen this result in the following way.

Corollary 7. For all $\delta>0$, there exists $0<c<1$ so that for all sufficiently large integers $n$, all but $c^{n}(n-1)$ ! cyclic permutations $\pi \in C_{n}$ admit $\frac{1}{12}\left(\begin{array}{c}n \\ 2\end{array}\right)(1 \pm \delta)$ stretching pairs.

Proof of Corollary 7. Let $\delta>0$ be given. Set $k=4$ and $\mu=\delta / 2$, and let $0<c_{1}<1$ be the constant guaranteed by Theorem 1. Define $c$ to be any constant satisfying $c_{1}<c<1$, and let $n$ be sufficiently large. For an $n$-cycle $\pi \in C_{n}$, write $\sigma(\pi)$ for the number of stretching pairs of $\pi$, and write $\sigma^{\prime}(\pi)$ for the number of stretching pairs $1 \leqslant \pi(i)<i<j<\pi(j) \neq n$. Note that $\sigma^{\prime}(\pi) \leqslant \sigma(\pi) \leqslant \sigma^{\prime}(\pi)+n$, since if $1 \leqslant \pi(i)<i<j<\pi(j)=n$, then $j=\pi^{-1}(n)$ is fixed and there are at most $j-1 \leqslant n$ choices for $i$. Note, moreover, that it follows 
from (25) that, for $p=\phi(\pi) \in S_{n-1}, \sigma^{\prime}(\pi)=f_{21-34}(p)+f_{34-21}(p)$. Theorem 1 ensures that all but $2 c_{1}^{n-1}(n-1) !<c^{n}(n-1)$ ! permutations $p \in S_{n-1}$ satisfy

$$
f_{21-34}(p)=(1 \pm \mu) \frac{1}{4 !}\left(\begin{array}{c}
n-1 \\
2
\end{array}\right) \quad \text { and } \quad f_{34-21}(p)=(1 \pm \mu) \frac{1}{4 !}\left(\begin{array}{c}
n-1 \\
2
\end{array}\right) .
$$

For each such permutation $p \in S_{n-1}$, the corresponding $n$-cycle $\pi=\phi^{-1}(p) \in C_{n}$ satisfies

$$
\begin{aligned}
\sigma(\pi) & =(1 \pm \mu) \frac{1}{4 !}\left(\begin{array}{c}
n-1 \\
2
\end{array}\right)+(1 \pm \mu) \frac{1}{4 !}\left(\begin{array}{c}
n-1 \\
2
\end{array}\right) \pm n \\
& =(1 \pm \mu \pm o(1)) \frac{1}{12}\left(\begin{array}{l}
n \\
2
\end{array}\right)=(1 \pm \delta) \frac{1}{12}\left(\begin{array}{l}
n \\
2
\end{array}\right),
\end{aligned}
$$

which proves Corollary 7.

\section{$5 \quad$ Proof of Lemma 6}

Fix $\zeta>0$ and integer $q \geqslant 1$. Define auxiliary constant

$$
\zeta_{0}=\zeta / 4 \text {. }
$$

Define $s_{0}=s_{0}\left(q, \zeta_{0}\right)$ to be the least integer $s$ for which

$$
(s)_{q} \geqslant\left(1-2 \zeta_{0}\right) s^{q}
$$

Let $s \geqslant s_{0}$ be given. Define

$$
t_{0}=\left\lceil 4 q 8^{q} s^{2 q} \zeta_{0}^{-2}\right\rceil
$$

Let integer $t \geqslant t_{0}$ be given. Define

$$
c_{0}=\frac{\zeta_{0}^{2}}{3 q t 2^{q+3} s^{q}} .
$$

Let $n$ be a sufficiently large integer, and fix $\left(\mathbf{i}_{0}, j_{0}\right) \in([s])_{q} \times[t]$. We prove

all but $\exp \left\{-2 c_{0} n\right\} n$ ! permutations $\pi \in S_{n}$ are $\left(\mathbf{i}_{0}, j_{0}, \zeta, q\right)$-typical w.r.t. $\left(\boldsymbol{P}_{s}, \boldsymbol{P}_{t}\right)$.

Applying (33) to all $(\mathbf{i}, j) \in([s])_{q} \times[t]$ and noting $s^{q} t \exp \left\{-2 c_{0} n\right\}<\exp \left\{-c_{0} n\right\}$ yields Lemma 6.

We now outline our approach for proving (33) (and reduce the $\hat{\ell}$ notation in (10) to $\ell)$. Define equivalence relation $\sim$ on $E_{j_{0}}: \ell \sim \ell^{\prime} \Longleftrightarrow q \mid\left(\ell-\ell^{\prime}\right)$. Thus, for an integer $0 \leqslant r<q$, we may write

$$
E_{j_{0}}^{(r)}=\left\{\ell \in E_{j_{0}}: \ell \sim\left(j_{0}-1\right)\left\lfloor\frac{n}{t}\right\rfloor+1+r\right\} \quad \text { so that } \quad E_{j}=E_{j}^{(0)} \cup \cdots \cup E_{j}^{(q-1)}
$$

is a partition. A key observation for later in the proof (cf. Claim 8) will be that

$$
[\ell, \ell+1-q] \cap\left[\ell^{\prime}, \ell^{\prime}+q-1\right]=\emptyset \quad \text { whenever } \ell \neq \ell^{\prime} \in E_{j}^{(r)} .
$$


For some final notation, we shall write, for a permutation $\pi \in S_{n}$,

$$
E_{\mathbf{i}_{0} j_{0}}^{(r)}(\pi)=E_{\mathbf{i}_{0} j_{0}}(\pi) \cap E_{j_{0}}^{(r)} \quad \text { so that } \quad E_{\mathbf{i}_{0} j_{0}}(\pi)=E_{\mathbf{i}_{0} j_{0}}^{(0)}(\pi) \cup \cdots \cup E_{\mathbf{i}_{0} j_{0}}^{(q-1)}(\pi)
$$

is a partition. We shall prove that, for a fixed $0 \leqslant r<q$,

$$
\text { all but } \exp \left\{-3 c_{0} n\right\} n ! \text { permutations } \pi \in S_{n} \text { satisfy that }\left|E_{\mathbf{i}_{0} j_{0}}^{(r)}(\pi)\right| \geqslant(1-\zeta) \frac{1}{q(s)_{q}}\left|E_{j_{0}}\right| \text {. }
$$

Note that (37) implies (33) since then all but $q \exp \left\{-3 c_{0} n\right\} n !<\exp \left\{-2 c_{0} n\right\} n$ ! many $\pi \in S_{n}$ satisfy

$$
\left|E_{\mathbf{i}_{0} j_{0}}(\pi)\right| \stackrel{(36)}{=} \sum_{r=0}^{q-1}\left|E_{\mathbf{i}_{0} j_{0}}^{(r)}(\pi)\right| \geqslant(1-\zeta) \frac{1}{(s)_{q}}\left|E_{j_{0}}\right| .
$$

To prove (37), let $\pi \in S_{n}$ be chosen uniformly at random. Then, $Y=Y_{\mathbf{i}_{0} j_{0}}^{(r)}=\left|E_{\mathbf{i}_{0} j_{0}}^{(r)}(\pi)\right|$ is a random variable whose mean we evaluate. To that end, recall from (10) that for an element $\ell \in E_{j_{0}}$ to be an element of $E_{\mathbf{i}_{0} j_{0}}(\pi)$, we require that $\ell \leqslant j_{0}\lfloor n / t\rfloor-q+1$, where we will write $n_{t}=\lfloor n / t\rfloor$ and $n_{s}=\lfloor n / s\rfloor$. As such, delete the last $q-1$ elements from $E_{j_{0}}$, and write

$$
\begin{aligned}
\tilde{E}_{j_{0}} \stackrel{\text { def }}{=}\left[\left(j_{0}-1\right) n_{t}+1, j_{0} n_{t}-q+1\right], \quad \tilde{E}_{j_{0}}^{(r)}=E_{j_{0}}^{(r)} \cap \tilde{E}_{j_{0}}, \\
\text { and } n_{t, q} \stackrel{\text { def }}{=}\left|\tilde{E}_{j_{0}}^{(r)}\right|=\left\lfloor\frac{n_{t}-q+1}{q}\right\rfloor=\left\lfloor\frac{n_{t}+1}{q}\right\rfloor-1 .
\end{aligned}
$$

Now, for $\ell \in \tilde{E}_{j_{0}}^{(r)}$, define indicator random variable $Y_{\ell}$ by $\left(\right.$ cf. $\left.\mathbf{i}_{0}=\left(i_{1}, \ldots, i_{q}\right)\right)$

$$
\begin{gathered}
Y_{\ell}=\left\{\begin{array}{c}
1 \\
\text { if } \pi(\ell+m-1) \in I_{i_{m}} \forall m \in[q], \\
0
\end{array} \quad \Longrightarrow \quad Y=\sum\left\{Y_{\ell}: \ell \in \tilde{E}_{j_{0}}^{(r)}\right\}\right. \\
\text { so that } \quad \mathbb{E}\left[Y_{\ell}\right]=\frac{(n-q) ! \prod_{m=1}^{q}\left|I_{i_{m}}\right|}{n !}=\frac{n_{s}^{q}}{(n)_{q}} \Longrightarrow \mathbb{E}[Y]=\frac{\left|\tilde{E}_{j_{0}}^{(r)}\right| \prod_{m=1}^{q}\left|I_{i_{m}}\right|}{(n)_{q}}=\frac{n_{s}^{q} n_{t, q}}{(n)_{q}} .
\end{gathered}
$$

Following the method of Bernstein for the Chernoff inequality (cf. [10]), for $u=\log (1-$ $\left.\zeta_{0}\right)=\log _{e}\left(1-\zeta_{0}\right)$, the Markov inequality implies

$$
\begin{aligned}
& \mathbb{P}\left[Y \leqslant \mathbb{E}[Y]\left(1-\zeta_{0}\right)\right]=\mathbb{P}\left[e^{u Y} \geqslant \exp \left\{u \mathbb{E}[Y]\left(1-\zeta_{0}\right)\right\}\right] \\
& \leqslant \exp \left\{-u \mathbb{E}[Y]\left(1-\zeta_{0}\right)\right\} \mathbb{E}\left[e^{u Y}\right] \stackrel{(39)}{=} \exp \left\{-u \frac{n_{s}^{q} n_{t, q}}{(n)_{q}}\left(1-\zeta_{0}\right)\right\} \mathbb{E}\left[e^{u Y}\right] .
\end{aligned}
$$

While we do not have mutual independence among the $Y_{\ell}$ 's, we will prove the following.

\section{Claim 8.}

$$
\begin{aligned}
& \mathbb{E}\left[e^{u Y}\right]=\mathbb{E}\left[\prod_{\ell \in \tilde{E}_{j_{0}}^{(r)}} e^{u Y_{\ell}}\right] \leqslant\left(1+q \frac{(4 s)^{q}}{t}\right)^{n_{t, q}} \prod_{\ell \in \tilde{E}_{j_{0}}^{(r)}} \mathbb{E}\left[e^{u Y_{\ell}}\right] \\
& \stackrel{(39)}{=}\left(\left(1+q \frac{(4 s)^{q}}{t}\right)\left(1+\frac{n_{s}^{q}}{(n)_{q}}\left(e^{u}-1\right)\right)\right)^{n_{t, q}} \leqslant \exp \left\{n_{t, q}\left(q \frac{(4 s)^{q}}{t}+\frac{n_{s}^{q}}{(n)_{q}}\left(e^{u}-1\right)\right)\right\} .
\end{aligned}
$$


We shall defer the proof of Claim 8 in order first to finish the proof of (37).

Applying Claim 8 to (40), together with the Taylor series bound $-u\left(1-\zeta_{0}\right)+e^{u}-1 \leqslant$ $-\zeta_{0}^{2} / 2$

$$
\begin{aligned}
\mathbb{P}[Y \leqslant & \left.\mathbb{E}[Y]\left(1-\zeta_{0}\right)\right] \leqslant \exp \left\{n_{t, q}\left(q \frac{(4 s)^{q}}{t}+\frac{n_{s}^{q}}{(n)_{q}}\left(-u\left(1-\zeta_{0}\right)+e^{u}-1\right)\right)\right\} \\
& \leqslant \exp \left\{n_{t, q}\left(q \frac{(4 s)^{q}}{t}-\frac{\zeta_{0}^{2} n_{s}^{q}}{2(n)_{q}}\right)\right\} \leqslant \exp \left\{n_{t, q}\left(q \frac{(4 s)^{q}}{t}-\frac{\zeta_{0}^{2}}{2}\left(\frac{n_{s}}{n}\right)^{q}\right)\right\} \\
& \leqslant \exp \left\{n_{t, q}\left(q \frac{(4 s)^{q}}{t}-\frac{\zeta_{0}^{2}}{2^{q+1} s^{q}}\right)\right\} \quad\left(\text { since } n_{s}=\lfloor n / s\rfloor \geqslant n /(2 s)\right) \\
& \stackrel{(31)}{\leqslant} \exp \left\{n_{t, q}\left(-\frac{\zeta_{0}^{2}}{2^{q+2} s^{q}}\right)\right\} \leqslant \exp \left\{-\left(\frac{\zeta_{0}^{2}}{q t 2^{q+3} s^{q}}\right) n\right\} \stackrel{(32)}{=} \exp \left\{-3 c_{0} n\right\} .
\end{aligned}
$$

(The last inequality above follows from $n_{t, q}=\left\lfloor\left(n_{t}+1\right) / q\right\rfloor-1 \geqslant n /(2 t q)$.) In other words, with probability $1-\exp \left\{-3 c_{0} n\right\}$, the randomly chosen permutation $\pi \in S_{n}$ satisfies

$$
\begin{aligned}
Y & =\left|E_{\mathbf{i}_{0} j_{0}}^{(r)}(\pi)\right| \geqslant \mathbb{E}[Y]\left(1-\zeta_{0}\right) \stackrel{(39)}{=} \frac{n_{s}^{q} n_{t, q}}{(n)_{q}}\left(1-\zeta_{0}\right) \stackrel{(38)}{=}\left(1-\zeta_{0}\right)(1-o(1)) \frac{1}{q s^{q}}\left\lfloor\frac{n}{t}\right\rfloor \\
& \stackrel{(30)}{\geqslant}\left(1-2 \zeta_{0}\right)^{2} \frac{1}{q(s)_{q}}\left\lfloor\frac{n}{t}\right\rfloor \geqslant\left(1-4 \zeta_{0}\right) \frac{1}{q(s)_{q}}\left\lfloor\frac{n}{t}\right\rfloor \stackrel{(29)}{=}(1-\zeta) \frac{1}{q(s)_{q}}\left\lfloor\frac{n}{t}\right\rfloor .
\end{aligned}
$$

\subsection{Proof of Claim 8}

Write $\tilde{E}_{j_{0}}^{(r)}$ as $\ell_{1}<\cdots<\ell_{n_{t, q}}$ (cf. (38)) so that

$$
\mathbb{E}\left[\prod_{\ell \in \tilde{E}_{j_{0}}^{(r)}} e^{u Y_{\ell}}\right]=\sum_{\left(y_{1}, \ldots, y_{n_{t, q}}\right) \in\{0,1\}^{n_{t, q}}} \mathbb{P}\left[\bigwedge_{i=1}^{n_{t, q}} Y_{\ell_{i}}=y_{i}\right] \prod_{i=1}^{n_{t, q}} e^{u y_{i}}
$$

Fix $\left(y_{1}, \ldots, y_{n_{t, q}}\right) \in\{0,1\}^{n_{t, q}}$ so that

$$
\mathbb{P}\left[\bigwedge_{i=1}^{n_{t, q}} Y_{\ell_{i}}=y_{i}\right]=\mathbb{P}\left[Y_{\ell_{n, q}}=y_{n_{t, q}} \mid \bigwedge_{j=1}^{n_{t, q}-1} Y_{\ell_{j}}=y_{j}\right] \cdot \mathbb{P}\left[\bigwedge_{j=1}^{n_{t, q}-1} Y_{\ell_{j}}=y_{j}\right]
$$

We claim that

$$
\mathbb{P}\left[Y_{\ell_{n_{t, q}}}=y_{n_{t, q}} \mid \bigwedge_{j=1}^{n_{t, q}-1} Y_{\ell_{j}}=y_{j}\right]=\mathbb{P}\left[Y_{\ell_{n_{t, q}}}=y_{n_{t, q}}\right]\left(1 \pm q \frac{(4 s)^{q}}{t}\right) .
$$

If so, iteratively applying (42) to (41) yields Claim 8. 
To see (42), recall the observation in (35). Thus,

$$
\begin{aligned}
\mathbb{P}\left[Y_{\ell_{t, q}}=1 \mid \bigwedge_{j=1}^{n_{t, q}-1} Y_{\ell_{j}}=y_{j}\right] & \leqslant \frac{\left(n-q n_{t, q}\right) ! \prod_{m=1}^{q}\left|I_{i_{m}}\right|}{\left(n-q\left(n_{t, q}-1\right)\right) !}=\frac{n_{s}^{q}}{\left(n-q n_{t, q}+q\right)_{q}}, \text { and } \\
\mathbb{P}\left[Y_{\ell_{t, q}}=1 \mid \bigwedge_{j=1}^{n_{t, q}-1} Y_{\ell_{j}}=y_{j}\right] & \geqslant \frac{\left(n-q n_{t, q}\right) ! \prod_{m=1}^{q}\left(\left|I_{i_{m}}\right|-q\left(n_{t, q}-1\right)\right)}{\left(n-q\left(n_{t, q}-1\right)\right) !} \\
& =\frac{\left(n_{s}-q n_{t, q}+q\right)^{q}}{\left(n-q n_{t, q}+q\right)_{q}} .
\end{aligned}
$$

For the upper bound, we use (39) (and $q n_{t, q} \leqslant n_{t}$ (cf. (38)) to infer

$$
\begin{aligned}
& \frac{n_{s}^{q}}{\left(n-q n_{t, q}+q\right)_{q}}=\mathbb{P}\left[Y_{\ell_{n_{t, q}}}=1\right] \cdot \frac{(n)_{q}}{\left(n-q n_{t, q}+q\right)_{q}} \leqslant \mathbb{P}\left[Y_{\ell_{n_{t, q}}}=1\right]\left(\frac{n}{n-n_{t}}\right)^{q} \\
& \leqslant \mathbb{P}\left[Y_{\ell_{n_{t, q}}}=1\right]\left(1-\frac{1}{t}\right)^{-q} \leqslant \mathbb{P}\left[Y_{\ell_{n_{t, q}}}=1\right]\left(1+\frac{2}{t}\right)^{q} \leqslant \mathbb{P}\left[Y_{\ell_{t, q}}=1\right]\left(1+q \frac{4^{q}}{t}\right) .
\end{aligned}
$$

For the lower bound, we similarly infer

$$
\begin{aligned}
\frac{\left(n_{s}-q n_{t, q}+q\right)^{q}}{\left(n-q n_{t, q}+q\right)_{q}} & \geqslant \frac{\left(n_{s}-q n_{t, q}\right)^{q}}{(n)_{q}}=\mathbb{P}\left[Y_{\ell_{n_{t, q}}}=1\right]\left(\frac{n_{s}-q n_{t, q}}{n_{s}}\right)^{q} \\
& \geqslant \mathbb{P}\left[Y_{\ell_{n_{t, q}}}=1\right]\left(\frac{n_{s}-n_{t}}{n_{s}}\right)^{q} \geqslant \mathbb{P}\left[Y_{\ell_{n_{t, q}}}=1\right]\left(1-2 \frac{s}{t}\right)^{q} \\
& \geqslant \mathbb{P}\left[Y_{\ell_{n_{t, q}}}=1\right]\left(1-q \frac{(4 s)^{q}}{t}\right) .
\end{aligned}
$$

This proves (42) when $y_{n, t}=1$. Otherwise, (using $\mathbb{P}\left[Y_{\ell_{n_{t, q}}}=1\right] \leqslant 1 / 2 \leqslant \mathbb{P}\left[Y_{\ell_{n_{t, q}}}=0\right]$ ) we have

$$
\begin{aligned}
\mathbb{P}\left[Y_{\ell_{n, q}}=0 \mid \bigwedge_{j=1}^{n_{t, q}-1} Y_{\ell_{j}}=y_{j}\right] & =1-\mathbb{P}\left[Y_{\ell_{t, q}}=1 \mid \bigwedge_{j=1}^{n_{t, q}-1} Y_{\ell_{j}}=y_{j}\right] \\
& =\mathbb{P}\left[Y_{\ell_{n_{t, q}}}=0\right]\left(1 \pm q \frac{(4 s)^{q}}{t}\right)
\end{aligned}
$$

where we used $\mathbb{P}\left[Y_{\ell_{n_{t, q}}}=1\right] \leqslant 1 / 2 \leqslant \mathbb{P}\left[Y_{\ell_{n_{t, q}}}=0\right]$.

\section{Acknowledgements}

We would like to thank the Referee for their careful reading of our work, and for helpful suggestions leading to an improved exposition. 


\section{References}

[1] Alon, N., Friedgut, E., On the number of permutations avoiding a given pattern, J. Combin. Theory, Ser. A 89 (2000), no. 1, 133-140.

[2] Babson, E., Steingrímsson, E., Generalized permutation patterns and a classificiation of the Mahonian statistics, Sém. Lothar. Combin. 44, Art. B44b (2000).

[3] Block, L., Coppel, W., Dynamics in One Dimension, Lecture Notes in Mathematics, Vol 1513, Springer-Verlag, Berlin, 1992.

[4] Bóna, M., Exact enumeration of 1342-avoiding permutations: A close link with labeled trees and planar maps, J. Combin. Theory Ser. A 80 (1997), 257-272.

[5] Bóna, M., The solution of a conjecture of Stanley and Wilf for all layered patterns, J. Combin. Theory Ser. A 85 (1999), no. 1, 96-104.

[6] Claesson, A., Generalized pattern avoidance, European J. Combin. 22 (2001), 961973.

[7] Cooper, J., Quasirandom permutations, J. Combin. Theory. Ser. A 106 (2004), 123142.

[8] Elizalde, S., Asymptotic enumeration of permutations avoiding generalized patterns, Adv. in Appl. Math. 36 (2006), 2851-2869.

[9] Elizalde, S., Noy, M., Consecutive patterns in permutations, Adv. in Appl. Math. 30 (2003), 110-125.

[10] Janson, S., Łuczak, T., Ruciński, A., Random Graphs, Wiley, New York (2000).

[11] Klazar, M., The Füredi-Hajnal conjecture implies the Stanley-Wilf conjecture, Formal Power Series and Algebraic Combinatorics (D. Krob, A.A. Mikhalev, and A.V. Mikhalev, eds.), Springer, Berlin, 2000, 250-255.

[12] Lundberg, E., Almost all orbit types imply period-3, Topol. and its Appl., 154, (2007), 2741-2744.

[13] Marcus, A., Tardos, G., Excluded permutation matrices and the Stanely-Wilf conjecture, J. Combin. Theory Ser. A 107 (2004), no. 1, 153-160.

[14] Sharkovsky, A.N., Coexistence of cycles of a continuous map of a line into itself, Ukrain. Mat. Zh. 16 (1964), 61-71 (Russian); English translation, Internat. J. Bifur. Chaos Appl. Sci. Engrg. 5 (1995), 1263-1273. 